\title{
Field investigation of Love waves in near-surface seismology
}

\author{
Robert Eslick ${ }^{1}$, Georgios Tsoflias ${ }^{1}$, and Don Steeples ${ }^{1}$
}

\begin{abstract}
We examine subsurface conditions and survey parameters suitable for successful exploitation of Love waves in nearsurface investigations. Love-wave generation requires the existence of a low shear-velocity surface layer. We examined the minimum thickness of the near-surface layer necessary to generate and record usable Love-wave data sets in the frequency range of 5-50 Hz. We acquired field data on a hillside with flat-lying limestone and shale layers that allowed for the direct testing of varying overburden thicknesses as well as varying acquisition geometry. The resulting seismic records and dispersion images were analyzed, and the Love-wave dispersion relation for two layers was examined analytically. We concluded through theoretical and field data analysis that a minimum thickness of $1 \mathrm{~m}$ of low-velocity material is needed to record usable data in the frequency range of interest in near-surface Love-wave surveys. The results of this study indicate that existing guidelines for Rayleigh-wave data acquisition, such as receiver interval and line length, are also applicable to Love-wave data acquisition.
\end{abstract}

\section{INTRODUCTION}

Surface-wave methods are a subset of near-surface seismic techniques. First introduced for near-surface applications in the $1950 \mathrm{~s}$ (Jones, 1958), surface-wave methods became viable along with more conventional near-surface seismic techniques, i.e., reflection and refraction, in the early 1980s (Steeples, 2005). Since that time, surface-wave methods have improved and expanded from the spectral analysis of surface waves (SASW) (Nazarian et al., 1983) to multichannel analysis of surface waves (MASW) (Park et al., 1999); the latter includes procedures for both passive and active sources (Park et al., 2007) as well as methods of utilizing higher-mode energy (Xia et al., 2000a).

To date, surface-wave techniques have focused mostly on the acquisition and inversion of Rayleigh-wave data. A strong foundation of theoretical and field studies has proven these methods reliable and useful in many geotechnical and environmental applications (Xia et al., 2000b; Miller et al., 1999a; Miller et al., 1999b). Very limited work on the use of Love waves has been reported in the literature (Lee and McMechan, 1992; Li, 1997).

Love waves are seldom used in near-surface investigations because of the relative difficulty in systematically producing a shearwave signal and because they require horizontal geophones. Love waves typically are considered an unwanted signal in near-surface shear-wave reflection surveys (Miller et al., 2001). At early times on seismograms collected with horizontal geophones, Love waves routinely dominate the seismic record and appear in the same phase-velocity range as reflections of interest. With increasing capability and decreasing cost of technology, it is becoming more viable to design shear-wave or multicomponent surveys in the near-surface.

Potentially, Love waves can yield shear-wave velocity profiles with little change to the Rayleigh-wave procedure. Zeng et al. (2007) and Song et al. (1989) show that Love waves generally are more sensitive to S-wave velocity changes and layer thickness changes than Rayleigh waves. An inherent difference is that Love-wave methods estimate horizontal shear-wave velocity, whereas Rayleigh-wave methods estimate vertical shear-wave velocity. Joint inversion of Love and Rayleigh data may yield information about subsurface anisotropy (Safani et al., 2005), such as the presence of fractures.

Extensive literature about Love waves, including the mechanics of propagation and dispersion, comes from earthquake seismology and theoretical physics (e.g., Sezawa and Kanai, 1937; Anderson, 1962; Drake, 1980; Kelly, 1983; Kim, 1992; Guzina and Madyarov, 2005). However, Love-wave surveys in the near-surface have been used and reported on only a few occasions (Jones, 1958; Song et al., 1989; Safani et al., 2005). The utility of the method and acquisition guidelines have not been investigated thoroughly. Several studies addressing these issues have been completed for Rayleigh waves (Beaty and Schmitt, 2003; Zhang et al., 2004). As surface-wave techniques continue to become attractive alternatives to other invasive and geophysical methods for near-surface site characterization, it is important to understand their applicability.

Our objective is to determine the limiting thickness for a low-velocity layer in order to record a usable Love-wave data set in near-

Manuscript received by the Editor 17 May 2007; revised manuscript received 5 September 2007; published online 1 May 2008.

${ }^{1}$ University of Kansas, Lawrence, Kansas, U.S.A.E-mail: rocyes@yahoo.com; tsoflias@ku.edu; don@ku.edu (C) 2008 Society of Exploration Geophysicists. All rights reserved. 
surface seismology. By recordable, we mean that Love waves can be observed in the seismic record; by usable, we mean that the record can yield a well-formed dispersion image. We create the images from multichannel records with the process used by Park et al. (1998) for Rayleigh waves. Acquisition parameters for Love-wave surveys follow Rayleigh-wave acquisition guidelines described in the MASW method (Park et al., 1999).

\section{THEORETICAL CONSIDERATIONS}

Surface waves travel mostly within one seismic wavelength of the earth's surface. They spread two-dimensionally and undergo energy decay at a rate of $1 / r$, where $r$ is the radial distance from the source. Body waves spread three-dimensionally, and their energy decays at $1 / r^{2}$. Rayleigh waves result from the interference of P- and SVwaves below a free surface. Their particle motion is retrograde elliptical and is confined to a nearly vertical plane in the direction of propagation. Love waves consist of only interfering SH-wave energy and have particle motion transverse to the direction of propagation. Rayleigh waves can exist in a half-space, although they are not dispersive if it is homogeneous and isotropic. Love waves are guided waves that are totally internally reflected. They require that a low-

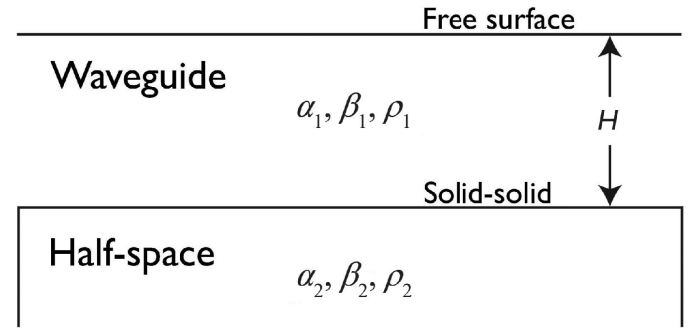

Figure 1. Two-layer model. Medium 1 is the waveguide; medium 2 is the half-space. The properties of each medium are described by their P-and S-wave velocities $\alpha$ and $\beta$, respectively, and density $\rho$. The thickness of the waveguide is $H$.

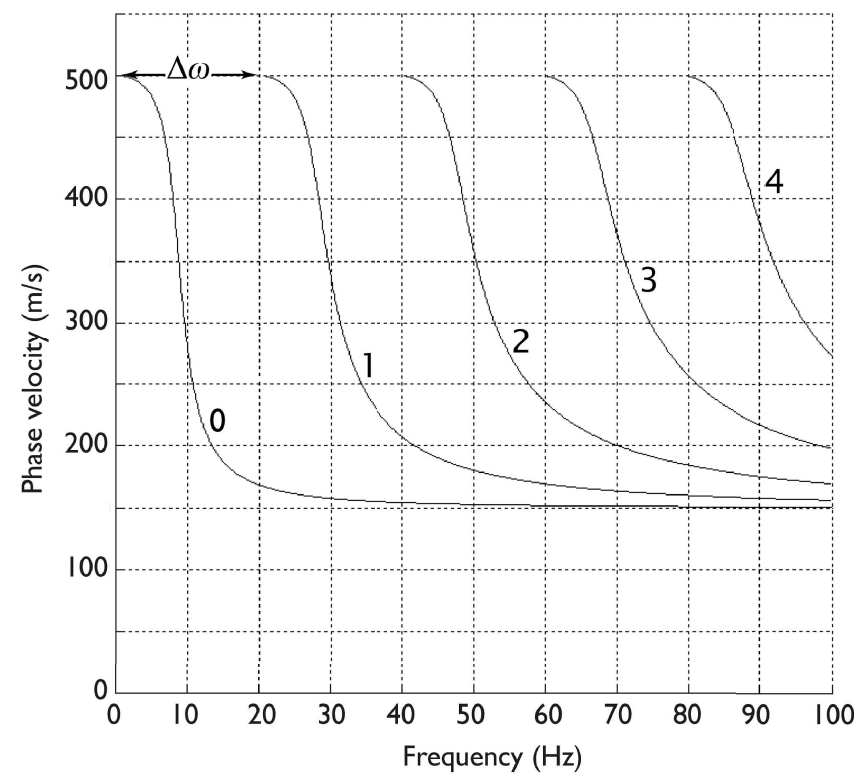

Figure 2. Theoretical dispersion curves for the model in Figure 1. The modes are numbered from the fundamental mode (0) to the fourth-higher mode (4). velocity layer (or waveguide) of some thickness $H$ be present (see Figure 1).

One form of the Love-wave dispersion relation for the two-layer case is given by Aki and Richards (2002) as

$$
\tan \left(\frac{\omega H}{c_{x}} \sqrt{\frac{c_{x}^{2}}{\beta_{1}^{2}}-1}\right)=\frac{\mu_{2} \sqrt{1-\frac{c_{x}^{2}}{\beta_{2}^{2}}}}{\mu_{1} \sqrt{\frac{c_{x}^{2}}{\beta_{1}^{2}}-1}},
$$

where propagation is in the $x$-direction; $\omega$ is angular frequency; $c_{x}$ is phase velocity; $\beta_{1}, \beta_{2}, \mu_{1}$, and $\mu_{2}$, are the shear-wave velocities and shear-moduli of the waveguide and half-space, respectively; and $H$ is the thickness of the waveguide.

We can see by examining this relation that Love-wave dispersion for horizontal layers is independent of $P$-wave velocity $\alpha$; given the relation $c_{x}=\omega / \kappa_{x}$, there are corresponding wavenumber $\kappa$ and phase velocity pairs that satisfy the relation for any given frequency. The solution with the lowest phase velocity is the fundamental mode; other solutions constitute higher modes. The solution to the relation must also satisfy $\beta_{1} \leq c_{x} \leq \beta_{2}$. This implies the phase velocities of low frequencies will approach the shear-wave velocity of the half-space $\beta_{2}$, and at high frequencies Love-wave phase velocities will approach the velocity of the waveguide $\beta_{1}$.

We evaluated equation 1 with typical near-surface parameters $\left(\beta_{1}\right.$ $=150 \mathrm{~m} / \mathrm{s}, \rho_{1}=1600 \mathrm{~kg} / \mathrm{m}^{3}, \beta_{2}=500 \mathrm{~m} / \mathrm{s}, \rho_{2}=1800 \mathrm{~kg} / \mathrm{m}^{3}$, and $H$ $=4 \mathrm{~m}$ ) and plotted the dispersion curves for this model, including higher modes, in Figure 2. Our analytical models agree with published results from Pelton (2005) and are further verified by replicating results from Stein and Wysession (2003) and Aki and Richards (2002). Multiple frequency/phase-velocity pairs satisfy equation 1 , which gives rise to higher modes. The separation between the modes is given by the cutoff frequency $\Delta \omega$ (Stein and Wysession, 2003):

$$
\Delta \omega=\frac{\pi}{H} \frac{\left(\beta_{1} \beta_{2}\right)}{\sqrt{\beta_{2}^{2}-\beta_{1}^{2}}} .
$$

We show the effect of changing $H$, when only the fundamental mode is considered, in Figure 3. As $H$ approaches zero m, the disper-

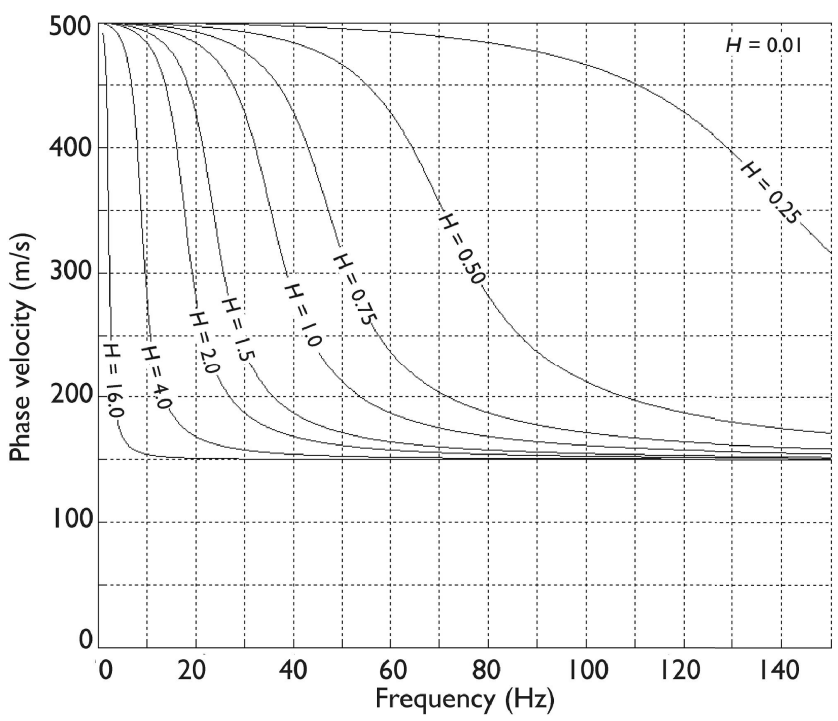

Figure 3. Theoretical fundamental mode dispersion curves for the model in Figure 1 as a function of waveguide thickness $H$. 
sion curve extends well beyond the frequency range obtainable in the field (commonly $<50 \mathrm{~Hz}$ ). As $H$ increases, the dispersion curve drops into the frequency range of interest; when $H$ is greater than 1 $\mathrm{m}$, the entire curve can be observed from 5 to $100 \mathrm{~Hz}$. As $H$ increases beyond $1 \mathrm{~m}$, the slope of the dispersion curve becomes steeper and the frequency range at which $c_{x}$ approaches $\beta_{2}$ becomes increasingly narrow.

Figure 4 illustrates the effect of $H$ on the formation of highermode energy. As $H$ increases, $\Delta \omega$ decreases such that at greater thicknesses the separation between the fundamental mode and higher modes decreases. Smaller values of $H$ give the fundamental mode a wider frequency range over which to exist before there is the possibility of higher mode contamination, i.e., small waveguide thicknesses are favorable in terms of avoiding higher-mode contamination.

We also examined the effect of velocity contrast between the lowvelocity waveguide and the underlying half-space using equation 1. Figure 5a shows a plot where $H$ and $\beta_{1}$ are held constant at $4 \mathrm{~m}$ and $150 \mathrm{~m} / \mathrm{s}$, respectively, and where $\beta_{2}$ varies from 1.01 to 10 times $\beta_{1}$. Maintaining constant waveguide velocity while changing $\beta_{2}$ does not significantly change the range of frequencies at which $c_{x}$ approaches $\beta_{1}$ or $\beta_{2}$, but it does change the slope of the dispersion curve between the minimum and maximum values. As the slope of the dispersion curve becomes increasingly steep (or as the velocity contrast becomes increasingly large), the frequency band over which it occurs becomes increasingly narrow and more difficult to record. Varying the waveguide velocity, i.e., contouring $\beta_{1}$, and holding $\beta_{2}$ constant (Figure 5b) exhibits broader changes in the range of frequencies at which $c_{x}$ approaches $\beta_{1}$ or $\beta_{2}$ as well as in the slope of the dispersion curve.

Phase velocity ultimately controls the wavelengths in the recorded data. Wavelength $\lambda$ is related to the phase velocity and frequency $f$ by $\lambda=c_{x} / f$. Recording long wavelengths necessitates longer receiver lines, which increases the subsurface area being averaged and can introduce far-field effects during dispersion-curve extraction and inversion. In high-velocity environments, wavelengths can

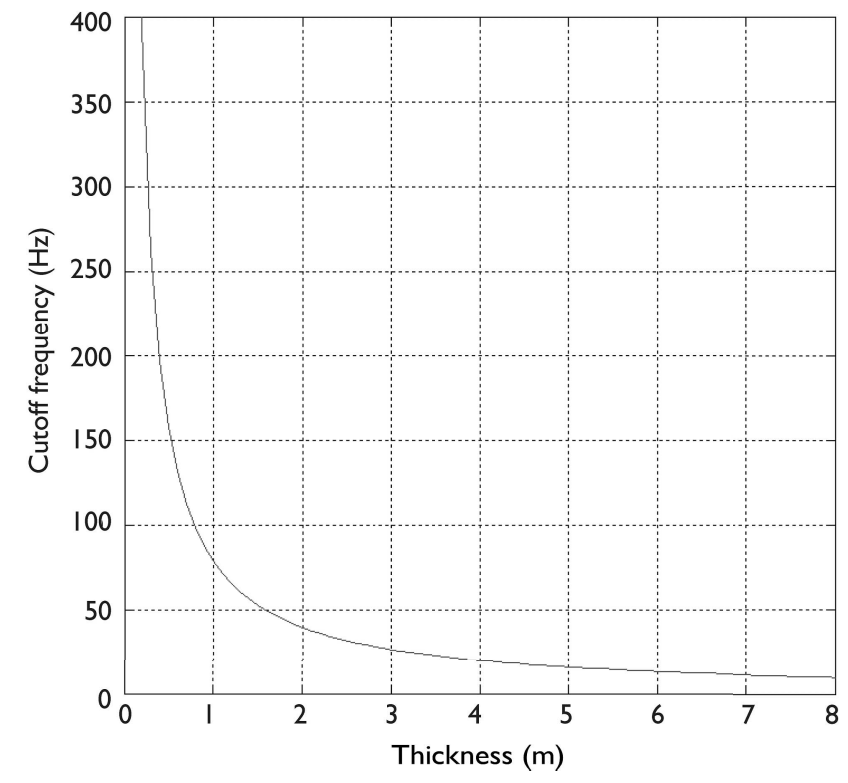

Figure 4. Cutoff frequency for the model in Figure 1 as a function of waveguide thickness $H$. lengthen and at some point become impractical for near-surface Love-wave surveys.

It is important to consider these relations because they have significant implications when acquiring and interpreting Love-wave data.

\section{FIELD METHOD}

A field site over a road cut south of Clinton Lake dam in Douglas County, Kansas, allowed for the direct testing of the effect of $H$ on the ability to collect Love-wave data in the field. For the current study and based on a priori information, the site is parameterized with two layers. The top layer consists of unconsolidated soils and eroded shale that form a hill that slopes at $25^{\circ}$ to $35^{\circ}$ and has a maximum accessible thickness of $4 \mathrm{~m}$. It is underlain by the Plattsmouth Limestone, which has an exposed thickness of $5 \mathrm{~m}$ below the hillforming overburden. The limestone layer is laterally continuous over a large area and constitutes a horizontal layer. The limestone layer was assumed thick enough, relative to time and offset range of interest, to be considered a half-space for this study. The field site is

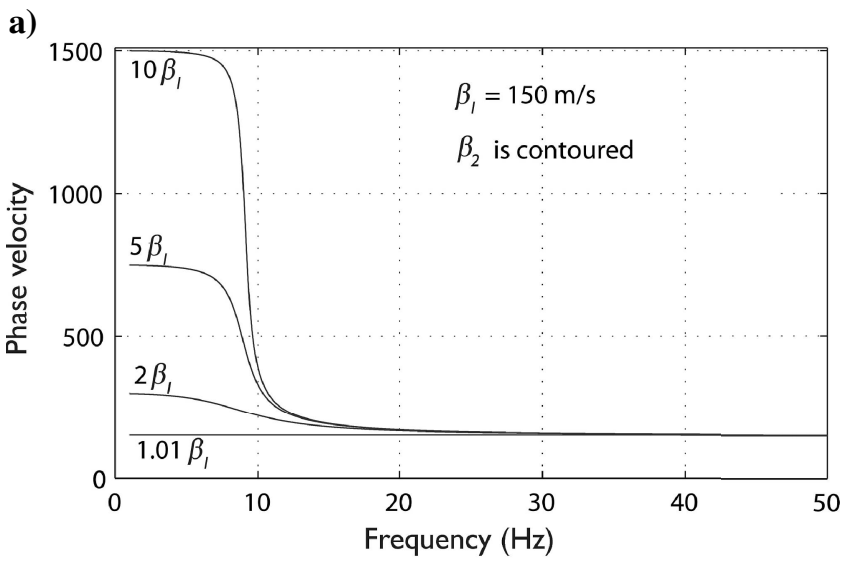

b)

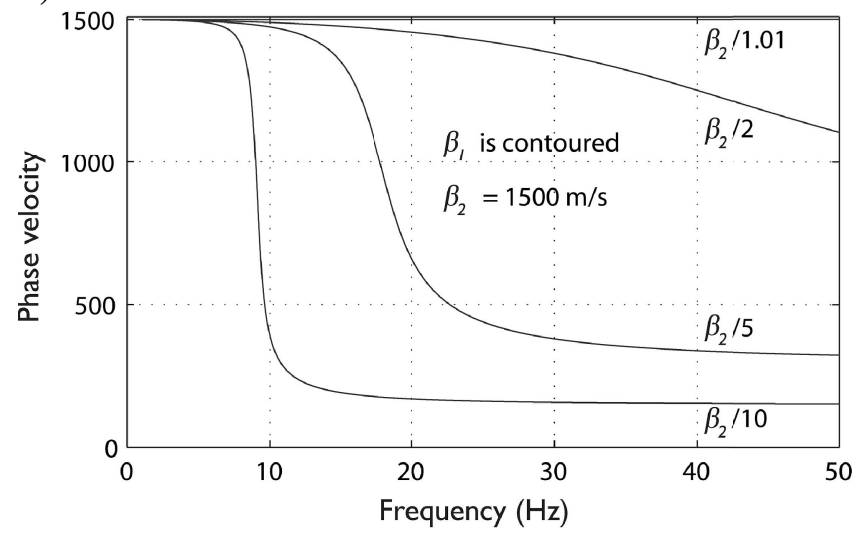

Figure 5. Theoretical dispersion curves for varying layer velocity contrast while maintaining waveguide thickness constant: (a) $\beta_{1}$ is held constant at $150 \mathrm{~m} / \mathrm{s}$ and $\beta_{2}$ varies from 1.01 to 10 times $\beta_{1}$. Maintaining constant waveguide velocity while changing $\beta_{2}$ does not significantly change the range of frequencies at which $c_{x}$ approaches $\beta_{1}$ or $\beta_{2}$. (b) Varying $\beta_{1}$ and holding $\beta_{2}$ constant exhibits broader changes in the range of frequencies at which $c_{x}$ approaches $\beta_{1}$ or $\beta_{2}$ as well as in the slope of the dispersion curve. 
an excellent analog to the model described by the dispersion relation given in equation 1.

We acquired several data sets at the field site to test the effect of $H$ and acquisition parameters on Love-wave data usability. Using the top of the limestone as the zero elevation datum $(H=0 \mathrm{~m})$, we placed receiver lines at $H=0.5,1.0,2.0$, and $4.0 \mathrm{~m}$ (see Figure 6). Each line consisted of $144,4.5-\mathrm{Hz}$ horizontal geophones at an interval of $0.25 \mathrm{~m}$. A 5-lb sledgehammer and a shear block were used as the seismic-energy source, which was located $0.25 \mathrm{~m}$ off the north end of each line.

\section{RESULTS AND DISCUSSION}

Raw, relative-amplitude seismograms acquired over varying waveguide thicknesses are shown in Figure 7. We can observe Love waves on all records; however, the offset range over which they extend and their frequency content change significantly with waveguide thickness $H$. At $H=0.5 \mathrm{~m}$, the relative energy of the Lovewave component of the record dissipates within $10 \mathrm{~m}$ of offset and the dominant frequency in the seismic record is roughly $35 \mathrm{~Hz}$. As $H$ increases, the offset range at which Love waves are present increases, and the dominant frequency decreases. At $H=4 \mathrm{~m}$, Love waves dominate the entire record from 0 to $36 \mathrm{~m}$ of offset, and the dominant frequency is $20 \mathrm{~Hz}$.

Analysis of the first arrivals gives $\beta_{1}$ and $\beta_{2}$ estimates of 100 and $700 \mathrm{~m} / \mathrm{s}$, respectively. Although the first arrival appears before the Love waves, it is difficult to interpret because of the low signal-tonoise ratio. However, dispersion images created from all records give reliable estimates in accordance with direct arrivals for $\beta_{1}$ of $100 \mathrm{~m} / \mathrm{s}$. Figures 8 and 9 show the dispersion images and interpreted dispersion curves for the lines at $H=1.0$ and $4.0 \mathrm{~m}$, respectively. The shape of these curves is consistent with the analytical models

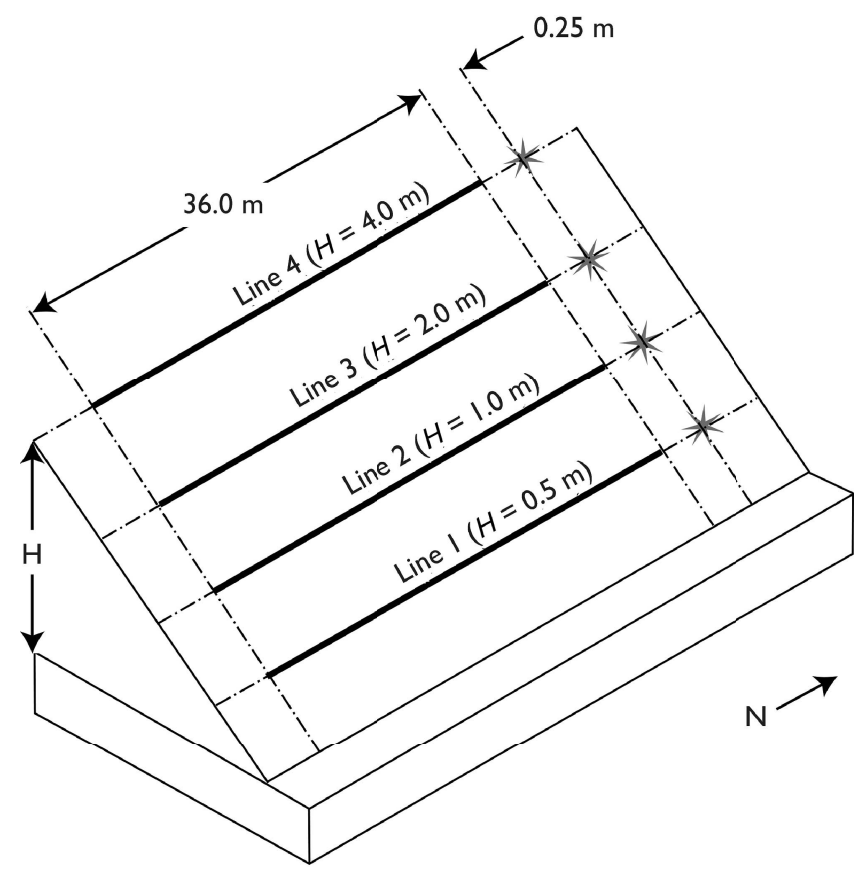

Figure 6. Field experiment setup. Receiver lines consisting of 144, $4.5-\mathrm{Hz}$ horizontal geophones at an interval of $0.25 \mathrm{~m}$ and a near-offset distance of $0.25 \mathrm{~m}$ were used to collect data at four different waveguide thicknesses.

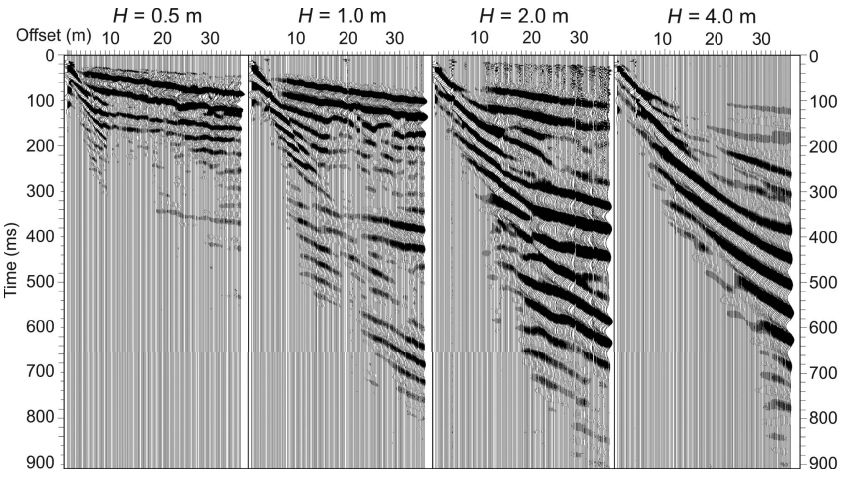

Figure 7. Raw, relative-amplitude seismic records corresponding to different waveguide thicknesses. Love-wave energy dissipates by $300 \mathrm{~ms}$ and $10 \mathrm{~m}$ of offset on the first record $(H=0.5 \mathrm{~m})$; on the last record $(H=4 \mathrm{~m})$, it persists over the entire range of offsets to about $850 \mathrm{~ms}$.

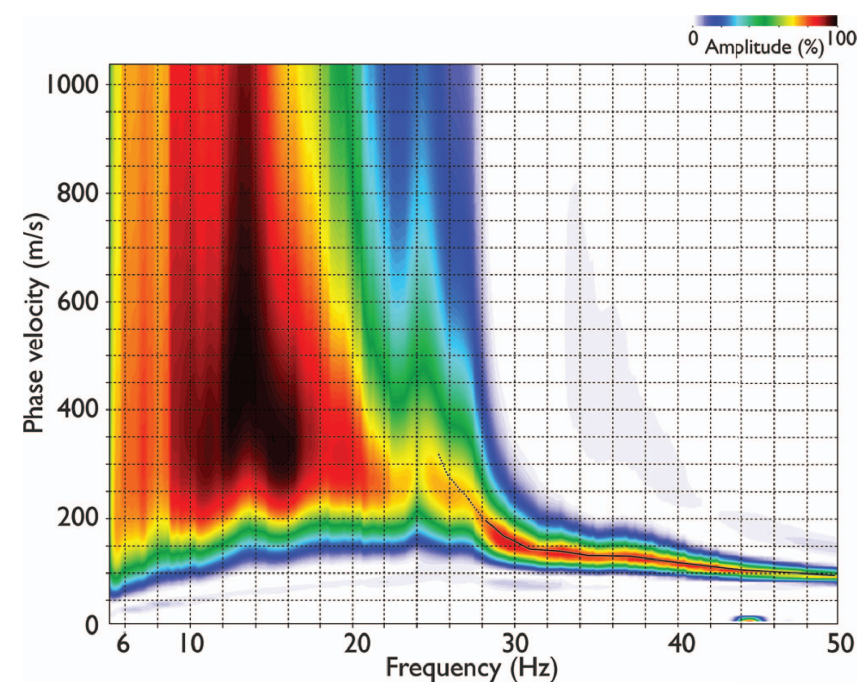

Figure 8. Dispersion image created from the receiver line at $H=$ $1.0 \mathrm{~m}$ with interpreted dispersion curve.

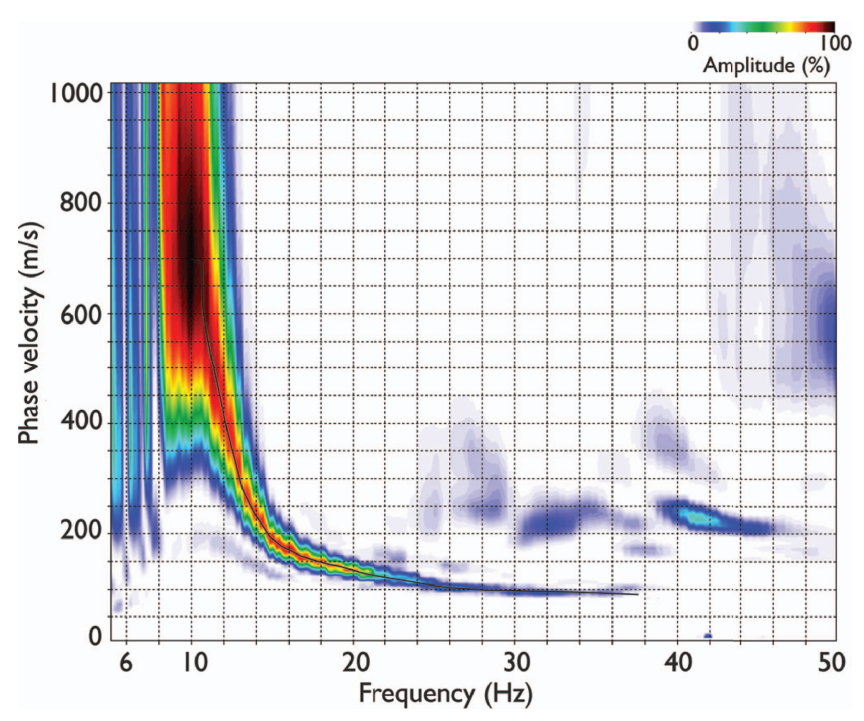

Figure 9. Dispersion image created from the receiver line at $H=$ $4.0 \mathrm{~m}$ with interpreted dispersion curve. 
(Figure 3), where at $H=1.0 \mathrm{~m}$ the curve has a gentler slope over a larger range of frequencies and at $H=4.0 \mathrm{~m}$ it becomes steeper over a smaller range of frequencies. Both of these curves are asymptotic to about $100 \mathrm{~m} / \mathrm{s}\left(\beta_{1}\right)$ at higher frequencies but become ambiguous at low frequencies, making $\beta_{2}$ difficult to interpret. From the dispersion curve at $H=4 \mathrm{~m}$ it is possible to independently interpret $\beta_{2}$ to be approximately $700 \mathrm{~m} / \mathrm{s}$ at $10 \mathrm{~Hz}$, which agrees with the refraction velocity. However, there is increasing uncertainty in frequencies less than $12 \mathrm{~Hz}$ where the phase velocity is $400 \mathrm{~m} / \mathrm{s}$, yielding a $\lambda$ of approximately $33 \mathrm{~m}$ - about the length of the receiver line.

The energy below $28 \mathrm{~Hz}$ in Figure 8 and below $12 \mathrm{~Hz}$ in Figure 9 is distorted because of misrepresented longer wavelengths relative to the receiver line length and other noise such as incidentally included body-wave energy, which obscures the upper boundary $\beta_{2}$. The layer thickness is a controlling factor in producing usable dispersion curves from Love-wave data at low frequencies. Figure 8 shows the dispersion curve from $H=1 \mathrm{~m}$; at a phase velocity of $200 \mathrm{~m} / \mathrm{s}$, the frequency is $28 \mathrm{~Hz}$, giving a $\lambda$ of approximately $7 \mathrm{~m}$. Even though the receiver line was five times that length, frequencies below $28 \mathrm{~Hz}$ are practically unusable.

Smaller waveguide thicknesses effectively restrain the lower frequencies or longer wavelengths from being generated. Theoretically, as shown in Figure 3, at thicknesses less than $1 \mathrm{~m}$, the frequency range over which the dispersion curve is asymptotic to $\beta_{2}$ becomes large and should be relatively clear. However, in field data collected where $H$ is less than $1 \mathrm{~m}$, the low-frequency component is missing and the range of offsets over which Love waves propagate becomes small. This essentially makes $\beta_{2}$ impossible to interpret from a dispersion curve created from data collected where the overburden thickness is less than about $1 \mathrm{~m}$.

\section{CONCLUSION}

Love-wave generation is dependent upon the presence of a slow shear-velocity waveguide below the free surface. The thickness of that layer directly relates to the ability to record usable data. We observed Love waves on seismic records where the thickness was as small as $0.25 \mathrm{~m}$, but they did not become usable until waveguide thickness was greater than $1 \mathrm{~m}$. The two-layer case study provides insight into the conditions that must exist for near-surface Lovewave surveys to be viable. If waveguide thickness is small, regardless of the velocity trend or layering within it, low-frequency energy is not generated within the layer and Love waves will not propagate to the offsets needed to produce the low-frequency component of the dispersion image. This cannot be overcome during data acquisition and constitutes the limiting case for which Love waves are applicable.

The acquisition-parameter guidelines for Rayleigh-wave data worked well for Love waves in this study. Any difference in field parameters between the two methods is accounted for by considering the corresponding shear-wave velocities and the resulting wavelengths. The most significant factors in surface-wave survey design are the highest and lowest velocity and frequency expected. The highest velocity and lowest frequency yield the longest wavelength, which should be approximately equal to the total line length, although there are other considerations such as the far-offset effect and the averaging effect of the inversion procedure. The near-offset distance should be greater than or equal to one-half of the longest wavelength to avoid the near-field effect. The lowest velocity and highest frequency yield the shortest wavelength and determine receiver spacing, which should be less than or equal to one-half of the shortest wavelength.

Literature addressing Love waves on a global scale and from a theoretical standpoint is widely available, and procedures for acquiring and utilizing Love-wave data are analogous to those for Rayleigh-wave data, which are well studied in the near-surface. However, continued research, including procedural, numerical, feasibility, and case studies, are needed so a basis for Love-wave surveys can be built and relied upon for near-surface site characterization.

\section{ACKNOWLEDGMENTS}

The authors thank Kwan Yee Cheng, Michael Christie, Gerard Czarnecki, Michael McGlashan, and Steve Sloan for assistance in the field. The authors also thank Julian Ivanov, Jianghai Xia, and Rick Miller at the Kansas Geological Survey for access to software and their helpful insight into surface waves. This research was supported by the Office of Science (BER), U.S. Department of Energy (DOE), grant DE-FG02-03ER63656. However, any opinions, conclusions, or recommendations expressed herein are those of the authors and do not necessarily reflect the views of the DOE.

\section{REFERENCES}

Aki, K., and P. G. Richards, 2002, Quantitative seismology: Theory and methods, 2nd ed.: University Science Books.

Anderson, D. L., 1962, Love wave dispersion in heterogeneous anisotropic media: Geophysics, 27, 445-454.

Beaty, K., and D. Schmitt, 2003, Repeatability of multimode Rayleigh-wave dispersion studies: Geophysics, 68, 782-790.

Drake, L. A., 1980, Love and Rayleigh waves in an irregular soil layer: Bulletin of the Seismological Society of America, 70, 571-582.

Guzina, B. B., and A. I. Madyarov, 2005, On the spectral analysis of Love waves: Bulletin of the Seismological Society of America, 95, 1150-1169.

Jones, R., 1958, In-situ measurement of the dynamic properties of soil by vibration methods: Geotechnique, 8, 1-21.

Kelly, K. R., 1983, Numerical study of Love wave propagation: Geophysics, 48, 833-853.

Kim, J. O., 1992, The effect of a viscous fluid on Love waves in a layered medium: Journal of the Acoustical Society of America, 91, 3099-3103.

Lee, H.-Z., and G. A. McMechan, 1992, Imaging of lateral inhomogeneities using Love wave data: 62nd Annual International Meeting, SEG, Expanded Abstracts, 992-994.

Li, X.-P., 1997, Elimination of higher modes in dispersive in-seam multimode Love waves: Geophysical Prospecting, 45, 945-961.

Miller, R. D., J. Xia, and C. B. Park, 2001, Love waves: A menace to shallow shear wave reflection surveying: 71st Annual International Meeting, SEG, Expanded Abstracts, 1337-1380.

Miller, R. D., J. Xia, C. B. Park, J. C. Davis, W. T. Shefchik, and L. Moore, 1999b, Seismic techniques to delineate dissolution features in the upper $1000 \mathrm{ft}$ at a power plant site: 69th Annual International Meeting, SEG, Expanded Abstracts, 492-495.

Miller, R. D., J. Xia, C. B. Park, and J. Ivanov, 1999a, Multichannel analysis of surfaces waves to map bedrock: Leading Edge, 18, 1392-1396.

Nazarian, S., K. Stokoe, and W. Hudson, 1983, Use of spectral analysis of surface waves method for determination of moduli and thickness of pavement systems: Transportation Research Record, 930, 38-45.

Park, C. B., R. D. Miller, and J. Xia, 1998, Imaging dispersion curves of surface waves on multichannel records: 68th Annual International Meeting, SEG, Expanded Abstracts, 1377-1380.

, 1999, Multichannel analysis of surface waves: Geophysics, 64 $800-808$

- 2007, Multichannel analysis of surface waves - Active and passive methods: The Leading Edge, 26, 60-64.

Pelton, J. R, 2005, Near-surface seismology: Wave propagation, in D. K. Butler, ed., Near-surface geophysics: SEG, 177-217.

Safani, J., A. O'Neill, T. Matsuoka, and Y. Sanada, 2005, Applications of Love wave dispersion for improved shear-wave velocity imaging: Journal of Engineering and Environmental Geophysics, 10, 135-150.

Sezawa, K., and K. Kanai, 1937, Relation between the thickness of a surface layer and the amplitudes of Love waves: Bulletin of the Earthquake Re- 
search Institute, University of Tokyo, 15, 577-581.

Song, Y., J. Castagna, R. Black, and R. Knapp, 1989, Sensitivity of near-surface shear-wave velocity determination from Rayleigh and Love waves: 59th Annual International Meeting, SEG, Expanded Abstracts, 509-512.

Steeples, D. W., 2005, Near-surface geophysics: 75 years of progress: The Leading Edge, 24, no. 10, S83-S85.

Stein, S., and M. Wysession, 2003, An introduction to seismology, earthquakes and earth structure: Blackwell Scientific Publications, Inc.

Xia, J., R. D. Miller, and C. B. Park, 2000a, Advantages of calculating shearwave velocity from surface waves with higher modes: 70th Annual International Meeting, SEG, Expanded Abstracts, 1295-1298.
Xia, J., R. D. Miller, C. B. Park, J. A. Hunter, and J. B. Harris, 2000b, Comparing shear-wave velocity profiles from MASW with borehole measurements in unconsolidated sediments, Fraser River Delta B. C., Canada: Journal of Environmental and Engineering Geophysics, 5, 1-13.

Zeng, C., J. Xia, Q. Liang, and C. Chen, 2007, Comparative analysis on sensitivities of Love and Rayleigh waves: 77th Annual International Meeting, SEG, Expanded Abstracts, 1138-1141.

Zhang, S., L. Chan, and J. Xia, 2004, The selection of field acquisition parameters for dispersion images from multichannel surface wave data: Pure and Applied Geophysics, 161, 185-201. 